\title{
Melgar y Olmedo
}

La causa de la Emancipación Americana tiene manifiesta expresión literaria en el Perú. Por supuesto que ella está especialmente contenida por las proclamas revolucionarias y por la obra de tendencia polemista que desde la "Carta" del jesuíta arequipeño Vizcardo ha de concluir en "Las Cartas Políticas" de José Faustino Sánchez Carrión, donde en lenguaje claro y gallardo se exhibe ol ideal republicano frente a las tendencias monarquistas que tomaron cuerpo en el Perú. Aquella expresión va descle el descontento intelectualista hasta la preeisa enunciación del pensamiento liberal y revolucionario. Ya cuando las Cortes de Cádiz ofrecen una transitoria libertad y una tendencia a formar un solo gran estado español constitucional, el diario "El Peruano" opinaba que aquí entendíamos "por Patria", la "vasta extensión de las dos Américas"; y luego ante la reacción autocrática que provoda la guera ded emancipación, los

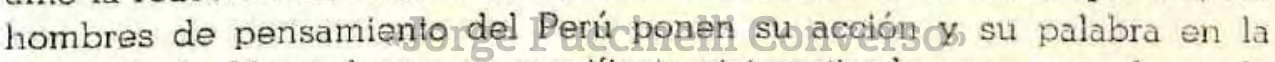
causa de la libertad que se manifiesta sistematizada y concretada en la citada producción de Sánchez Carrión, exponente del sentimiento libertario republicano, como Baquijano y Carrillo lo es de la insurgencia criolla y como Unánue de la afirmación nacional.

Al lado de la propaganda clandestina de los criollos rebeldes y de la musa popular que acompaña la acción libertadora y que está presente al lado de San Martín y Bolívar, entonando canciones revolucionarias con léxico típico y con influencia indígena, dos indudables poetas representan, más que otros, dos aspectos de la literatura emancipadora: la voz ín. tima y telúrica y la entonación grandielocuente. Uno se llama Melgar; el otro, Olmedo.

El fervor nacionalista, centro de la concepción filosófica naturalista y su secuela el individualismo, forman la base del movimiento romántico que ha de sistematizarse en los estudios literarios de Schlegel. Esas manifestaciones primarias adquieren excepcionales contornos en el paso de 
las formalidades clásicas de Andrés Chenier al sentimentalismo de su "Joven Cautiva"; y en las apasionadas manifestaciones de los jóvenes alemanes del "Sturm Und Drang". Es el romanticismo en gérmenes. Y en América, en el Perú, Melgar representa un anticipo del movimiento romántico, muchos años anles de su verdadera entrada oficial. En Melgar se presenta el sentido vernacular de los "Yaravies"; la apreciación de los factores naturaleza e individuo, dentro de una tabulación sentimental, con el ideal de la libertad y la refrendación del martirio jor esa misma causa.

Nacido en Arequipa, en 1791, Mariano Melgar, con señalada precosidad, leía a Cicerón - con largos períodos y con el calor de su fe repu. blicana-y lo traducía para sus compañeros de colegio cuando aún no había cumplido los 10 años. También en el propio Seminario de Sar Jerónimo hacía traducciones de Virgilio, con la manifestacion del agro que tenía eco y resonancia en las visiones del medio "chacarero" arequi-. peño. El famoso educador Chávez de la Rosa le confiere la primera tonsura y Mariano Melgar continúa en sus estudios de "Humanidades", dedicado por completo al perfecionamiento intelectual. Surge, entonces, ei amor a Silvia, y el consiguiente abandono de la carrera eclesiástica. Cuan do los padres de Melgar deciden enviarlo a Lima a estudiar leyes, brotan los primeros cantos apasionados ajenos a la formación intelectual y clasicista. El mar, motivo sólo decorativo para escenas de piratas, produce en Melgar el primer canto de su inmensidad y de su abandono, como notas esencialmente líricas. II'At Auter del Mar't dediça el poeta su angustia; y el apasionado Jegresa de Mollendo h Arequipa, dispuesto a desobedecer las órdenes paternas. Silvia, la propia amada, le pide que cumpla con aquellas y entonces vuelve a emprender el viaje a la capital.

"¿Por qué a verte volví, Silvia querida?

¡Ay triste! ¿para qué? ¡Para trocarse

mi dolor en más triste despedida!"

Vive en Lima en medio de la conjuración revolucionaria y ya, de antemano, está enrolado en la causa de la rebeldía y de la libertad. A ella dedicará más tarde una de sus odas.

La vuelta a Arequipa significa la decepción. Silvia había olvidado al amante y avasalladora surge la punzante nota:

"No nació la mujer para querida por esquiva, por falsa y por mudable......" 
El primer intento de doblegar la pasión se manifiesta en la lectura y traducción de los "Remedios de Amor" de Ovidio, que se convierten en "Arte de Olvidar". El segundo, el trabajo como agricultor en el valle de Majes; allí convive con peonadas indias y escucha las variantes mestizas del antiguo "harawi", donde se confunden las manifestaciones hispanas con la raíz indígena. Melgar inicia entonces la gran tarea de hacer una lírica nacional en castellano. Con el estribillo quechua traducido:
"Vuelve que ya no puedo
vivir sin tu cariño;
vuelve mi palomita,
vuelve a tu dulce nido"......

Melgar lleva adelante la temática desarrollando el tema del abandono, fundamental en la lírica precolombina, y crea el "yaravi":
"Mira que hay cazadores
que con afán maligno te pondrán en sus redes
mortales atractivos;
y cuando te hayan preso
te darán cruel martirio:
No sea que te cacen, huye de tal peligroca de Letras
"Jorge Puccinelli Converso»
"Vuelve mi palomita. vuelve a tu dulce nido".

Melgar ha pasado definitivamente al campo "romántico". La "urpillay", la palomita que no vuelve al nido, motivo-raíz de la lírica uqechua, es recogida, adaptada, a la poesía castellana, transformándose el "harawi" en el "yaraví" y ofreciendo la primera manifestación mestiza indo-hispana con categoría de tal. No importe que la dicción sea deficiente; que el verso no sea acabado, recortado.

La revolución contra la Corona española detenida por la esperanza de las cortes gaditanas, prende nuevaménte y el Sur peruano se levanta en armas poniendo como jefe y símbolo de la insurrección al Brigadier Pumacahua. Melgar decide luchar al lado de los patriotas, y aún entonces su poesía se llena de amoroso lamento: 


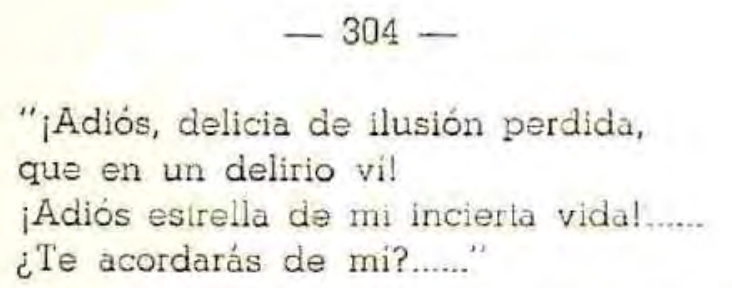

Alistado en Chuquibamba, vuelve a Árequipa a despedirse de sus padres. Las escenas de entonces han sido repetidas muchas veces pou los biografos de Melgar, quien está dispuesto a dejarse matar por silvià, la que desdeñó el amor del poeta:

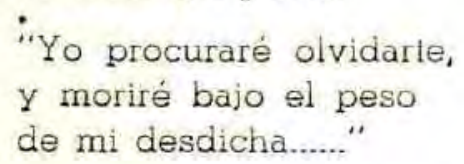

La influencia quechua deja sentirse a cada momento, en medio de las "Elegias" clásicas:

$$
\text { "Muerto yo, tú llorarás......" }
$$

Auditor de Guerra del Ejercilo Patriota, Melgar cae prisionero despues del desastre de Humachiri $y$ es condenado a muerte. Su valentía se manfiesta entonces en su deseo de mortr. En aquellas instantes la Patria y la Amada se confunden en IVelgar, como en el Danle se pueden confundir la Gracia y Beatriz. En la mañana del 12 de marzo de 1815, Mariano Melgar es fusilado, cuando apenas tenía 24 años.

La mayor parte de lá obra de este sinquiar pgeta nuestro se ha perdido, pues su hermana josela la quemo a indicacion del confesor que consideraba la poesia "derpelgarcfocolledireanterspor sensual y escéptico. Quedan, sin embargo, recogidas por tradición oral muchas de ellas y reproducidas en la Lira Arequipeña y otras publicaciones: cinco odas, cinco fâbulas, dos sonetos, cinco elegias, diez yaravies y su "carta" a Sil$\mathrm{via}_{i}$ en itálicus endecasílabos asonantados. También superviven algunas muestras de sus traducciones latinas. Pero sobre todo persiste la leyenda popular alrededor de su figura y de su obra que responde ya al concepto de lo peruano en su cabal sentido. $Y$ en todos los pueblos de nuestro territorio se repite alguna estrofa de sus yaravíes:

"Todo mi afecto puse en una ingrata y ella inconstante me llegó a olvidar.

Si así, si así se trata un afecto sincero, amor, amor no quiero, no quiero más amar"...... 
Melgar representa el nacionalismo literario en su cariño por la tierra, afirmación de las raíces populares de la literatura, y en la personificación de los ideales de libertad. Su obra se cumple íntegramente en el campo lírico; en los yaravíes y elegías amorosas y en las Odas compuestas "Al Autor del Mar" - "cerca del ancho mar"- "A la Libertad" o "Al Conde de Vista Florida".

También dentro de la lírica, pero en la manifestación exaltada de la Oda Heroica, se ha de desarrollar la figura literaria del otro gran poeta de la Emancipación. Juan José Remos señala que fué "el más alto portalira del ritmo heroico", según cita de Alberto Tauro: y añade que además tuvo el acierto de cantar al hombre "enmarcándolo en la luz de la propia tierra"; y que "junto al gigante de los hombres del Sur, Bolívar, destacó el coloso de sus montañas: los Andes".

José Joaquín Olmedo, nació en Guayaquil, perteneciente al Virreinato del Perú, en 1780; y a los 14 años estudiaba en el Convictorio Carolino, al lado de otras atormentadas estampas de la libertad. Profesor de Filosofía a los 20 años en aquel centro de Estudios, a los 30 era Maestro en San Marcos, después de haber obtenido los grados de Abogado y Doctor en Jurisprudencia. Junto con Morales Duarte, partió en 1810 a las Corles de Cádiz, representando à su región; y en 1816, envuelto en el círculo liberal, conjuraba en América contra el renacimiento de la autocracia española. Cuando Guayaquil proclama la Libertad, es Olmedo el Jefe Político de la Plaza Yojerce su función por dos años, hasta que Bolívar decreta su anexión a Colombia, y Olmedo, que defiende la causa tradicional peruanista de Guayaquil, viaja hadial-Gma después de protestar por aquella imperiosa determinación del Libertador. Se le ve figurar aquí como Diputado por Guayaquil al primer Congreso Constituyente y junto con el caudillo liberal Sánchez Carrión formar la Comisión que invita a Bolívar a terminar la obra emancipadora; más tarde es diplomático peruano acreditado en Londres hasta 1827, en que régresa a Guayaquil y en ocaso la Gran Colombia de Bolívar, Olmedo toma parte en la política del naciente estado ecuatoriano hasta 1847, en que fallece sin haber logrado la Presidencia de su país.

Ásí como Melgar camina hacia el romanticismo, Olmeda se afianza en su formación clásica. Producto de ella es indudablemente la obra fundamental de su genio poético -improductivo por la acción pública de sus últimos años - ya que la oda "A la Victoria de Junín" es un vaciamiento de los modelos helénicos al campo y a la acción americana. De allí que Bolívar en su carta considerara que del campo de Junín había hecho Olmedo escenario de combate troyano y de las figuras de los patriotas 
estampas de los legendarios personajes homéricos. "Si yo no fuese tan bueno y Ud. no fuese tan poeta, me avanzaría a creer que Ud. había querido hacer una parodia de la llíada con los héroes de nuestra pobre farsa".

Equivocaba, sin embargo, Bolívar. Olmedo había indudablemente recogido las lecturas greco-latinas, pero su estro no estaba inflamado por la poesía homérica - tersa y artistica en su cabal søntido- y si más bien había estereotipado el modelo de la Oda pindárica - postrera entonación alambicada del ditirambo-. Olmedo puso en juego los sentimientos y las visiones de América en armas contra la opresión y fué la suya poesía patriótica e inspirada y la más alta expresión literaria de la Emancipación Americana. .

No hay Antología Poética de habla castellana que no inserte aquellos trozos iniciales de su oda "A la Victoria de Junín", donde el poeta em plea la onomatopeya con facilidad y con efectivos resultados:
"El trueno horrendo que en fragor revienta y sordo retumbando/ se dilata
por la inflamada esfera,
al Dios anuncia que en el cielo impera.
$Y$ el rayo que en Junín rompe y ahuyenta
la hispana muchedumbre
que más feroz que nunca amenazaba
a sangrel $y$ fuega eterna servidumbre;
$y$ el canto de victoria
que enfecos mill discurre entsordeciendo
el hondo valle y enriscada cumbre,
proclaman a Bolívar en la tierra
árbitro de la paz y de la guerra"......

El adjetivo galopa siempre al lado de cada substantivo en un permanente deseo de dar énfasis a las oraciones y ya es "el alazán fogoso", la "nube tremenda", el "vivo reflejo de la gloria", "los duros opresores"; el "violento tropel de los guerreros". Los héroes patriotas están envuel. tos en una ola de vigorosa exaltación poética y de pronto con indudable "metier", Olmedo baja el tono - para volver a subirlo después en uns sucesión de cargas de caballerías y resonar de trompetas-y dice sua. vemente:

"¿Son esos los garzones delicados entre seda y aromas arrullados?......" 
Así también, buscando efectos, el poeta hace descender la sombra de Huayna Cápac sobre el campo ensangrentado de Junín y al anunciar la victoria de los libertadores y de magnificar las glorias del Imperio bajo la advocación del sol, pide nuevas campañas, tan duras como las libradas para la conquista de la paz, entonando un himno triunfal al trabajo.

Olmedo recuerda a su contemporáneo el español Quintana, vigoroso poeta de la escuela salmantina, inflamado de nacionalismo y de liberalismo, entonando Odas Heroicas por la acción popular contra Napoleón y haciendo del adjetivo ampuloso la base de su enérgica dicción: "El fiero Atila que a Occidente oprime". Pero Olmedo es más artista que Quintana; éste más reflexivo prepara su obra poética extrayéndola de una retórica donde elimina los galicismos y lucha contra la influencia afrancesada de la composición.

Olmedo es un poeta vigoroso; "lirismo bizarro y resonancia espléndida", dice Jiménez Borja. Todos nuestros críticos han subrayado ese viril entusiasmo, esa facilidad de la composición que desarrolla la batalla de Junín en ágiles expresiones y con elevada entonación donde el idioma edquiere belleza y resonancia. Olmedo es el gran cantor de la gloria bélica con el telón omnipotente de las cumbres andinas y puede perdonársele la ampulosidad y aún la intromisión del "deus ex machine" final que tanto se le ha censurado.

"El numen de Olmedo - dice Porras Barrenechea - sólo halla su correspondencia en la voz tremante de patria y plena de arranque tribunicio de Sánchez Carrión en el Congresó Constituyente, Gevantándose para sostener lúcidamente lá tedra deula divisiỏn Cellosepoderes o conjurar el espectro de la tiranía agazapado tras del poder unipersonal o cuando suena con chasquido de látigo en la prosa vibrante del "Solitario de Sayán, para desbaratar los planes monárquicos de Monteagudo, fustigando la adulación y el servilismo y haciendo el férvido elogio de la dignidad y la virtud republicanas".

\section{Bibliografía}

Al celebrarse este año el Centenario de la muerte de José Joaquín Olmedo, las naciones americanas, en particular los países bolivarianos, han testimoniado su afecto por el autor de. "La Oda a la Victoria de Junín" y nuevas e interesantes páginas se han escrito sobre su obra que tiene un resonante eco de libertad y un vívido ambiente andirio. La bibliografía sobre Olmedo se ha enriquecido. Podemos citar como interesante para una confrontación del juicio de la posteridad sobre este poeta peruano, la bibliografía existente en nuestra Biblioteca Nacional, relacionada directamente con el autor: 
Andrade Coello, Alejandro.-

Olmedo. (Motivos nacionales. Quito, 1911-27, tomo 1, págs. 97-120). Benites Vinneza, Leopoldo.-

El sentido humano en Olmedo. Letras del Ecuador. No 19-20, Dic: 1946-Feb. 1947; p. 3-6.

Castillo, Abel Romeo.-

Actuación constitucionalista de Olmedo. Letras del Ecuador. No 19-20. Dic. 1946-Feb. 1947, p. 3.

Documentos relacionados con los célebres ecuatorianos José Joaquín Olmedo y Vicente Rocafuerte. Boletín de la Academia Nacional de Historia. Quito. Ecuador. Ene-Jun. 1947, p. 116-128.

Castillo, Abel Romeo,-

Olmedo, el político (discurso parlamentario... con motivo del primer centenario de la revolución marciana...). Guayaquil, Imp. de la Universidad, 1946. Bibliografía al pie de algunas páginas.

Corpancho, Manuel Nicolás.

Poesías inéditas de Olmedo; apuntes bibliográficos para formar una edición más completa que las conocidas. México, Ed. del "Heraldo", 1862.

Espinosa Polit, Aurelio, S. I.-

Olmedo, el hombre públito. Boletín de la Academia Nacional de Historia. Quito. Ecuador. Ene-jun. 1947, No 69, p. 5-26.

Espinosa Polit, Aurelio S. I.

Olmedo y Horado? las dos "Agtualas". Cuenea-Ecuador, 1937. Olmedo, José joaquínołge Puccinelli Converso»

...Obras completas... Texto establecido, prólogo y notas por el Padre Aurelio Espinosa Polit. Quito. Ed. de la Casa de la Cultura Ecua toriana, 1945.

Olmedo, José loaquín.-

... Obras poéticas... Unica colección completa, revista y corr. por el autor y ordenada por J. M. G... París, Lib. Española y Americana de D. Ingo Boix y Cía., 1853.

Olmedo, José Joaquín.-

Poesías. Ed. corr. conforme a los manuscritos o primeras ediciones con notas, documentos y apuntes biográficos por Clemente Ballén. París, Garnier Hnos. 1815.

Olmedo, José Joaquín...

La Victoria de Junín, canto a Bolívar, $4^{\text {a }}$ ed. Guayaquil. Imp. Múnicipal, 1917. 
Olmedo, José Joaquín.-

La Victoria de Junín, canto a Bolívar, por J. J. Olmedo. París, 1826. Sánchez, Luis Alberto.-

Los poetas de la revolución. 3a conferencia leída el 22 de setiembre de 1919. Lima, 1919.

Sánchez, Luis Alberto.-

Los Poetas de la Colonia y de la Revolución, Edit. P.T.C.M. 1947. Tauro, Alberto.-

Elementos de Literatura Peruana. Edic. "Palabra". 1946. p. 60-63. Váscones, Francisco.-

Olmedo y sus obras; estudio histórico-crítico... Guayaquil, Lib. e Imp. Gutemberg, 1920.

La bibliografía sobre Melgar es menos nutrida y recientemente se ha logrado el nuevo perfil del poeta, alejándolo de las enconadas diatribas con que los escritores academistas cubrieron su personalidad. La obra de Melgar es interesante no sólo en cuanto a la iniciación del "yaravi", sino que representa un movimiento literario en conjunción con dos aspectos de gran envergadura universal. individuo y naturaleza, conjugados en el momento del liberalismo roussoniano. En la Biblioteca Nacional, encontramos:

Biblioteca de Cultura Peruana-París. 1938. Tomo XIII. Los Románticos. p. 15-27.

Camacho, Diego.-

El poeta Mariano Metgar, héroe y marir. Indice. Lima. Perú. No 28. Enero de 1946.

Dabadie, F. -

A travers l'Amérique du Sud... Paris, Ferdinand Sartorius, 1858. "Les curiosités de Lima. Les liméniennes. Les brigands du Pérou". p. 135-220. "Le poete des Andes: Melgar". p. 221-235.

García Bedoya, José Manuel.-

Tesis para optar el grado de bachiller en la Facultad de "Filosofía y Letras", que presentó ante el Concejo de la Universidad del Gran Padre San Agustín de Arequipa... Moquequa, Imp. "El Universo", 1891.

Melgar, Mariano.--

... Obras completas. Lima. Ed. Durán. Bibliografía de Melgar, por Pedro Durán Q. Prólogo de L. F. Xammar. 1944.

Melgar, Mariano.-

Poesías de Don Mariano Melgar. Publícalas D. Manuel Moscoso Melgar, dedicándolas a la juventud arequipeña, Lima. Nancy Tip. de G. Crépin-Leblond. 1878. 


\section{Melgar, Mariano.-}

Poesías selectas. La Literatura Peruana. Lima. 1923. No 5, Año I. Melgar, Mariano.-

Album del Centenario de Melgar. Arequipa. Imp. de "La Bolsa", 1891. Melgar, Mariano.-

El cancionero mistiano; selecta recopilación de canciones, valses, polkas, mazurcas, yaravies, jotas, etc., etc. 1月 ed. Arequipa. Tip. Quiroz, 1914.

Melgar, Mariano.-

Mistura para el bello sexo; canciones y yaravies, antiguos y modernos, $1^{\text {a }}$ serie. Arequipa. Imp. "La Bolsa", 1893.

Polar, Jorge.-

Nuestro Melgar. Ensayo biográfico. Arequipa 19..

San Cristóval, Evaristo.-

Poeta y héroe (Mariano Melgar). Lib. e Imp. Gil S. A., 1944. "Bibliografía": p. 63-67.

Sánchez, Luis Alberto.-

Ob. ya citada. p.

Tapia Olarte, Eulogio.-

La influencia clásica en Melgar. Revista Universitaria. Cuzco. № 79. 1940. p. 53-59. Además: EY Deber. Arequipa, 14 de agosto de 1947, p. 9. Tauro, Alberto.-

Ob. ya citada. p. $57-60$ ca de Letras

Urquieta, Mariano Lino.

Balbuceos parríticos. Fulín de 1900 ? Árequipa, Imp. "La Bolsa", 1900. Discursos en loor de Melgar y de San Martín.

Xammar, Luis Fabio.-

Romanticismo y libertad. Cultura Peruana. Vol. IV. No 16, p. 48-51;y El Deber. Arequipa, 10 de Feb. 1948. p. 7.

El primer romántico peruano. Lá Prensa. Buenos Aires. 30 de Enero de 1944. 